\title{
Evolução dos acidentes de trabalho na agropecuária, antes e após a criação da NR 31
}

Os trabalhadores que desempenham atividades na agropecuária estão constantemente expostos a uma diversidade de riscos e doença ocupacionais. A NR 31 é a norma regulamentadora que rege as regras para a saúde e segurança do trabalhador no meio rural, a qual passou a vigorar no país a partir de março de 2005. Desse modo, o objetivo do trabalho foi avaliar a evolução histórica dos acidentes de trabalho na agropecuária, antes e após a criação dessa norma regulamentadora. Para isso, foram avaliados os dados disponíveis no Anuário Estatístico de Acidentes de Trabalho (AEAT), no período de 1999 a 2017, para atividade econômica 'Agricultura, Pecuária e serviços relacionados'. Foram avaliados os acidentes de trabalho típico com CAT (Comunicação de Acidente do Trabalho) no Brasil e nos estados da região Sudeste. Além disso, foram analisados a evolução histórica da gravidade desses acidentes no mesmo período e locais citados acima, por meio dos dados relacionados à consequência dos acidentes. Pode-se observar com a análise realizada que após a criação da NR31 houve uma queda no número de acidentes de trabalho no meio rural do Brasil. Foi verificada essa mesma tendência nos estados da região sudeste, nos quais houve uma redução de $84 \%$ (Rio de Janeiro), 73\% (Espírito Santo), 69\% (São Paulo) e 61\% (Minas Gerais). Em relação ao estado de São Paulo é notável que, além da redução no número de acidentes, houve redução na gravidade dos mesmos. Nesse contexto, os resultados encontrados evidenciam uma redução geral de acidentes após a criação da NR31, no entanto, os números ainda são alarmantes, considerando que a maioria dos acidentes no campo não são registrados em órgãos oficiais, sendo subnotificados. Estudos como esse são relevantes para ressaltar a importância de se investir em ações preventivas relacionadas à saúde e integridade física dos trabalhadores.

Palavras-chave: Saúde e segurança do trabalho; Ambiente rural; Trabalhador rural; Riscos ocupacionais.

\section{Evolution of accidents at work in agropecuaria, before and after the creation of NR 31} health and safety of workers in rural areas, which came into force in the country as of March 2005. Thus, the aim of the work was to evaluate the historical evolutio of accidents work in agriculture, before and after the creation of this regulatory norm. For this, the data available in the Statistical Yearbook of Accidents at Work (AEAT), from 1999 to 2017, for economic activity 'Agriculture, Livestock and related services' were evaluated. Typical work accidents with CAT (Work Accident Communication) in Brazil and in the states of the Southeast region were evaluated. In addition, the historical evolution of the severity of these accidents in the same period and locations mentioned above was analyzed, using data related to the consequence of accidents. It can be seen from the analysis that after the creation of NR31 there was a drop in the number of work accidents in rural Brazil. This same trend was observed in the states of the southeast region, in which there was a reduction of $84 \%$ (Rio de Janeiro), $73 \%$ (Espírito Santo), 69\% (São Paulo) and 61\% (Minas Gerais). In relation to the state of São Paulo, it is notable that, in addition to the reduction in the number of accidents, there was a reduction in their severity. In this context, the results found show a general reduction in accidents after the creation of the NR31, however, the numbers are still alarming, considering that most accidents in the field are not registered in official bodies, being underreported. Studies like this are relevant to highlight the importance of investing in preventive actions related to workers' health and physical integrity.

Keywords: Health and safety; Rural environment; Rural worker; Occupational risks.

Topic: Políticas, Planejamento e Gestão de Sistemas e Serviços de Saúde

Reviewed anonymously in the process of blind peer
Received: 20/02/2020

Approved: 04/05/2020
Amanda Azarias Guimarães (iD)

Universidade Federal de Lavras, Brasil

http://lattes.cnpq.br/6423162579404456

http://orcid.org/0000-0002-1945-7356

amandaazarias@gmail.com

Tamires Galvão Tavares Pereira (ic

Universidade Federal de Lavras, Brasil

http://lattes.cnpq.br/2193131970787592

http://orcid.org/0000-0003-3423-6962

tamires florestal@hotmail.com

Hércules José Marzoque

Universidade Federal de Lavras, Brasi

http://lattes.cnpq.br/1794664336140572

http://orcid.org/0000-0001-7052-0659

hercules_marzoque@hotmail.com

\author{
Marcelo Linon Batista (D) \\ Universidade Federal de Lavras, Brasil \\ http://lattes.cnpq.br/1905350618721525 \\ http://orcid.org/0000-0002-7744-6049 \\ marclinon@yahoo.com.br \\ Douglas Lamounier Faria (iD) \\ Universidade Federal de Lavras, Brasil \\ http://lattes.cnpq.br/2575514568061593 \\ http://orcid.org/0000-0002-5405-8430 \\ douglas.lamounier@yahoo.com
}

Referencing this:

GUIMARÃES, A. A.; PEREIRA, T. G. T.; MARZOQUE, H. J.; BATISTA, M. L.; FARIA, D. L.. Evolução dos acidentes de trabalho na agropecuária, antes e após a criação da NR 31. Scire Salutis, v.10, n.2, p.67-72, 2020. DOI: http://doi.org/10.6008/CBPC2236-9600.2020.002.0009 


\section{INTRODUÇÃO}

Durante a Revolução Verde, entre as décadas de 1960 e 1970, iniciou a modernização do setor agropecuário brasileiro. Nesse período, com o intuito de aumentar a produtividade, foram introduzidas no campo novas técnicas de manejo, a mecanização agrícola, a utilização de agroquímicos e defensivos animais, entre outras inovações tecnológicas (SILVA et al., 2014).

Diante das mudanças ocorridas no processo produtivo, observou-se um aumento significativo dos riscos e da gravidade dos acidentes de trabalho no campo, além de ter proporcionado o surgimento de novos tipos de acidentes. A necessidade de aumentar a produção de alimentos, seguida pelo alto custo de produção, teve como consequência o prolongamento da jornada de trabalho, o que contribuiu para a ocorrência de mais acidentes (RODRIGUES et al., 1986).

Segundo a Organização Internacional do Trabalho (OIT), a agricultura é uma das atividades de maior índice de acidentes no mundo, equiparando à construção civil e mineração. As atividades desempenhadas pelos trabalhadores rurais são consideradas arriscadas e insalubres, deixando-os expostos a diversos tipos de riscos ambientais (químicos, físicos e biológicos), riscos de acidentes e riscos ergonômicos (ALMEIDA et al., 1995; SILVEIRA et al., 2005). As atividades desempenhadas no meio rural podem variar desde a limpeza do local de trabalho a preparação do solo, operação de máquinas, aplicação de agroquímicos (ACOSTA, 2015). O uso intensivo de agroquímicos na agricultura moderna, além de expor o trabalhador rural a sérios riscos à saúde, podem causar impactos ambientais e representar um risco à segurança alimentar da população (LOPES et al., 2018).

Antigamente, os acidentes no meio rural ocorriam quase que somente na utilização de ferramentas manuais. No entanto, com a utilização de tecnologias cada vez mais avançadas, as máquinas agrícolas, que são importantes para o aumento da produtividade no campo, têm sido a causa da maioria dos acidentes neste setor. Segundo Ambrosi et al. (2013), baseado em pesquisa realizada com trabalhadores rurais, observou-se que $45 \%$ dos acidentes ocorreram em máquinas agrícolas, seguido por acidentes com ferramentas manuais, correspondendo a $33 \%$.

Muitos dos acidentes que ocorrem nos ambientes de trabalho do setor agrícola podem provocar lesões que resultam na incapacidade temporária ou permanente e doenças ocupacionais (BRAUND et al., 2007). Essas lesões necessitam de tratamentos que geram custos elevados, sendo superiores em tempo e dinheiro quando relacionados aos custos necessários para a construção de um ambiente seguro, incluindo treinamento dos trabalhadores.

A consolidação das leis de trabalho ocorreu em 1943, por meio do Decreto Lei №5.452, de maio de 1943 (BRASIL, 1943). No entanto, foi com a modernização da agricultura, na década de 70, que surgiram normas reguladoras específicas para o trabalho rural, Lei no 5.889 de 8 de junho de 1973, na qual consta a definição de empregado e empregador rural. Na Constituição Federal de 1988, o trabalhador rural teve seus direitos equiparados aos direitos do trabalhador urbano, além de outras garantias individuais. A Norma Regulamentadora de no 31 (NR 31), aprovada pela portaria do Ministério do Trabalho e Emprego no 86, 03 
de março de 2005, refere-se à Segurança e Saúde no Trabalho na Agricultura, Pecuária, Silvicultura, Exploração Florestal e Aquicultura. Com a vigência dessa NR, foi revogada a Portaria do MTb no 3.067, de 14 de abril de 1988, na qual dispunha a aprovação das Normas Regulamentadoras Rurais - NRR do artigo 13 da Lei no 5.889, de 08 de junho de 1973, relativas à Segurança e Higiene do Trabalho Rural (BRASIL, 1988; 1973).

A NR 31 dá suporte para que seja possível aumentar a segurança e saúde do trabalhador no campo. No entanto, no Brasil, são escassos estudos que mostram o impacto da criação dessa NR, em números absolutos, nos acidentes de trabalho na agropecuária. Desse modo, considerando a importância dessa classe trabalhadora para o desenvolvimento econômico do país, objetivou-se avaliar a evolução histórica dos acidentes de trabalho no meio rural no período de 1999 a 2017, demonstrando os números de acidentes antes e depois da criação da NR 31.

\section{MATERIAIS E MÉTODOS}

O Anuário Estatístico de Acidentes de Trabalho (AEAT) disponibiliza os dados de acidentes de trabalho no período de 1999 a 2017. Desse modo, para a sistematização do número de acidentes de trabalho na agropecuária (ATA), no país e na região sudeste, foram utilizados os dados desse período, considerando a divisão do CNAE 95 e 2.0 - Agricultura, Pecuária e serviços relacionados. O número de acidentes típicos-com CAT são predominantes em relação aos outros tipos de acidentes (de trajeto e doenças do trabalho), por isso se optou por analisar somente os acidentes contabilizados dentro dessa classificação/situação.

Além disso, também foram sistematizados os ATA liquidados (no país e no Sudeste) de acordo com a consequência do acidente (Assistência Médica; Incapacidade por menos de 15 dias; Incapacidade por mais de 15 dias; Incapacidade permanente; Óbito). Os dados foram analisados por meio de estatística descritiva. Importante ressaltar, que no Brasil, a maioria dos ATA não são relatados às autoridades oficiais para registro, o que dificulta a obtenção de dados seguros sobre a frequência e gravidade destes acidentes.

\section{RESULTADOS E DISCUSSÃO}

O agronegócio corresponde a 21,7\% do PIB nacional (CEPEA, 2017), o que demonstra a importância desse setor para econômica do país. Essa alta representatividade no PIB, corresponde à atividade intensa de trabalhadores no setor. Mesmo assim o número de notificações de acidentes de trabalho na agropecuária em 2017 foi relativamente baixo quando comparado com o total de notificações no Brasil. De acordo com os dados divulgados pelo AEAT, o Brasil teve 340.229 notificações de acidentes de trabalho (típico-com CAT) em 2017, dos quais 3,6\% (12.292 acidentes) ocorreram na agricultura, pecuárias e serviços relacionados (Divisão CNAE). As notificações de acidentes típicos-com CAT neste setor econômico têm reduzido nos últimos anos, como pode ser observado na Figura 1. No entanto, ainda existe uma grande preocupação em relação a ocorrência de acidentes de trabalho e ambiental, por ser considerado um ambiente insalubre e de alto risco.

A maioria dos acidentes poderiam ser evitados, considerando que os principais motivos estão relacionados a condições inseguras (utilização de ferramentas defeituosas, máquinas sem dispositivos de segurança, falta de treinamento e capacitação), ato inseguro (excesso de confiança do trabalhador, não 
utilização dos Equipamentos de Proteção Individual, utilizar ferramentas de forma imprópria, exibicionismo) e o fator pessoal de insegurança que levam o trabalhador à prática do ato inseguro, independentemente da sua vontade (são fatores externos, próprios das características físicas e psicológicas de cada um, como tensão, estresse, insegurança, condições sociais, econômicas e financeiras). Este último fator foi considerado a principal causa de acidentes em máquinas agrícolas na região de Monte Carmelo/MG, segundo Alvarenga et al. (2017). Estes autores verificaram que a falta de atenção do operador correspondeu a $73 \%$ das causas dos acidentes ocorridos com máquinas agrícolas na região avaliada.

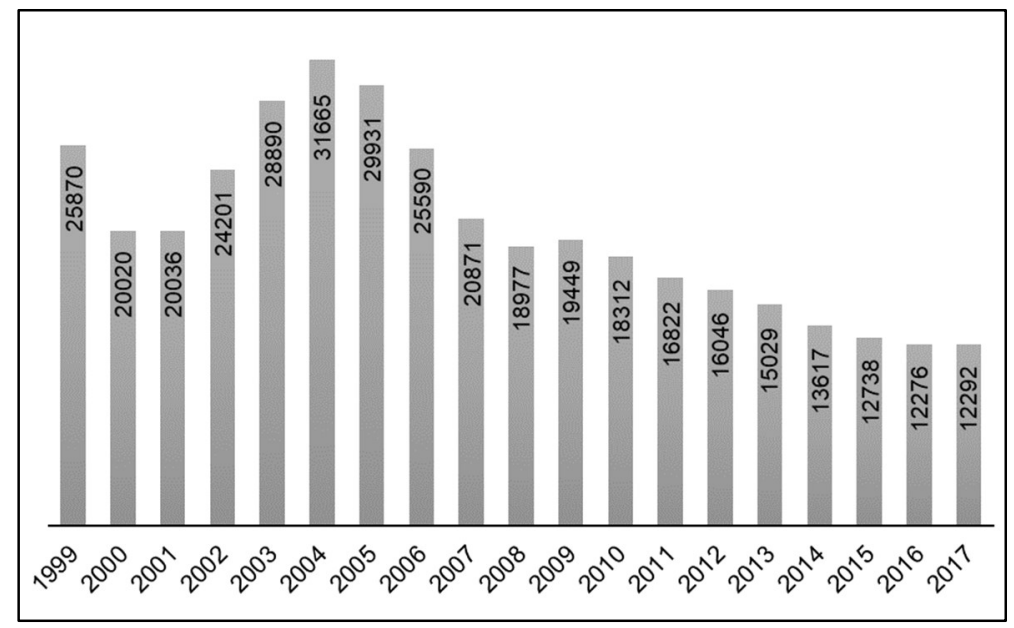

Figura 1: Evolução histórica dos acidentes de trabalho na agricultura, pecuária e serviços relacionados (Divisão CNAE) que ocorreram no Brasil entre 1999 a 2017 (Motivo/Situação: típico-com CAT).

A evolução histórica das notificações de acidentes de trabalho no meio rural, em todo Brasil, demonstrou a ocorrência de mais de 30.000 acidentes em 2004 (Figura 1), esse foi o máximo de acidentes registrados entre os anos de1999 e 2017. Dentre os estados da região sudeste, foi registrado o maior número de ATA em São Paulo, seguido por Minas Gerais (Figura 2).

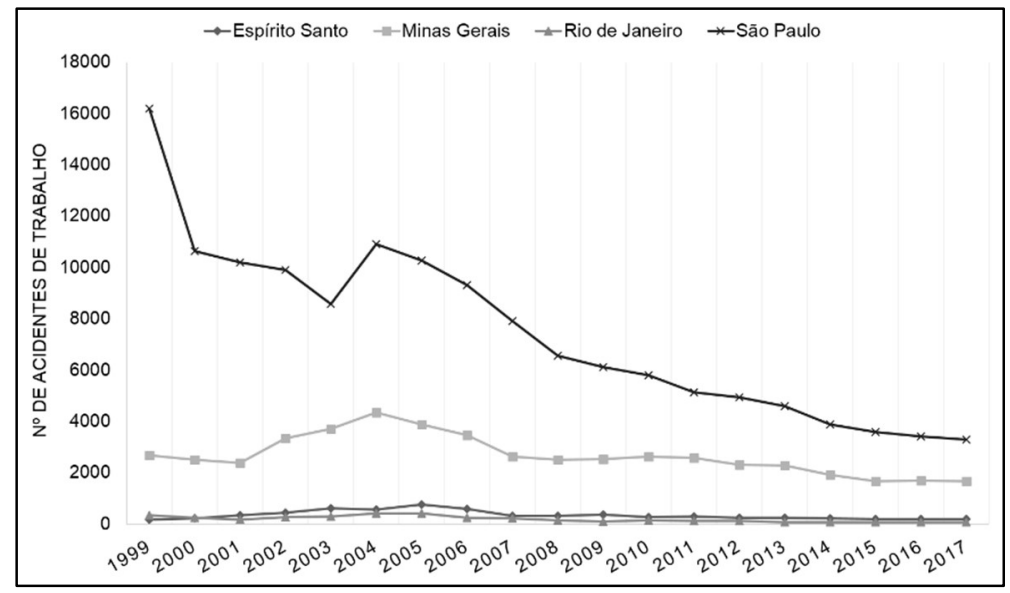

Figura 2: Evolução histórica dos acidentes de trabalho na agricultura, pecuária e serviços relacionados (Divisão CNAE) por UF, na região sudeste do Brasil, que ocorreram no período de 1999 a 2017 (Motivo/Situação: típico-com CAT).

Em SP o maior número de ATA registrados ocorreu em 1999, enquanto nos outros estados o maior número de acidentes ocorreu em 2004/2005 e o menor em 2017. Nesse período, foi observado uma redução de $84 \%$ dos acidentes no Rio de Janeiro, 73\% no Espírito Santo, 69\% em São Paulo e 61\% em Minas Gerais. 0 aumento crescente de acidentes observados entre 2002 e 2004 pode estar relacionado à aprovação do 
regulamento de inspeção do trabalho no final de 2002, por meio do decreto № 4.552, de 27 de dezembro de 2002 (BRASIL, 2002). Enquanto a queda que ocorreu nos anos seguintes pode estar relacionada com a promulgação da NR 31.

Quando foram avaliados os acidentes de trabalho de acordo com as consequências, no Brasil, verificou-se que há maior incidência de acidentes com afastamento por menos de 15 dias (Figura 3). Os acidentes com afastamento por mais de 15 dias reduziram significativamente nos últimos cinco anos, sendo registrados 8110 acidentes em 2014, enquanto em 2017 foram 2621 acidentes. Para acidentes com afastamento por mais de 15 dias, o trabalhador recebe auxílio-doença da previdência social, mediante perícia médica. Já as despesas de acidentes com afastamento de até 15 dias são de responsabilidade do empregador. Desse modo, a redução de acidentes com afastamento por mais de 15 dias corresponde também uma redução nos custos da previdência social.

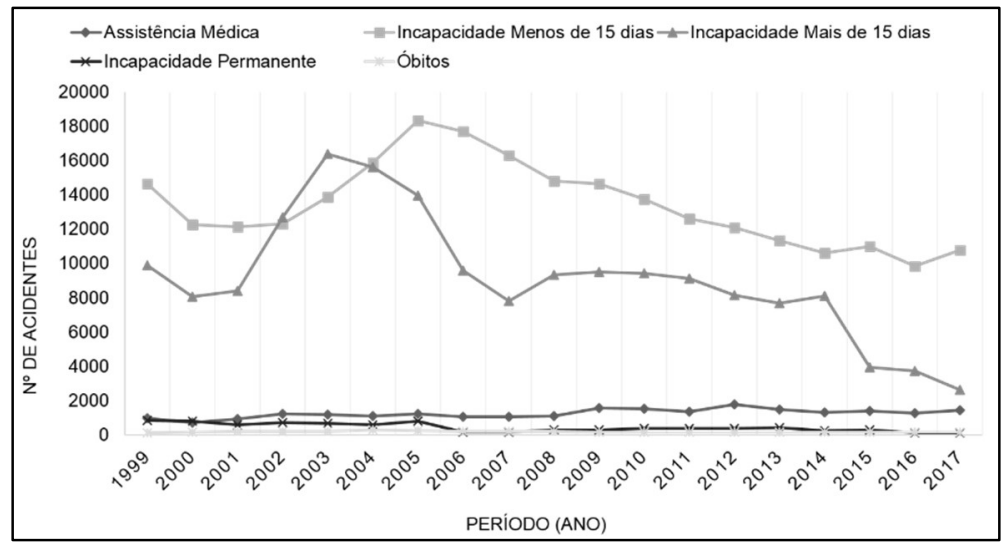

Figura 3: Evolução histórica dos acidentes de trabalho entre 1999 a 2017 de acordo com as consequências: Assistência Médica; Incapacidade - menos de 15 dias; Incapacidade - mais de 15 dias; Incapacidade Permanente; Óbitos.

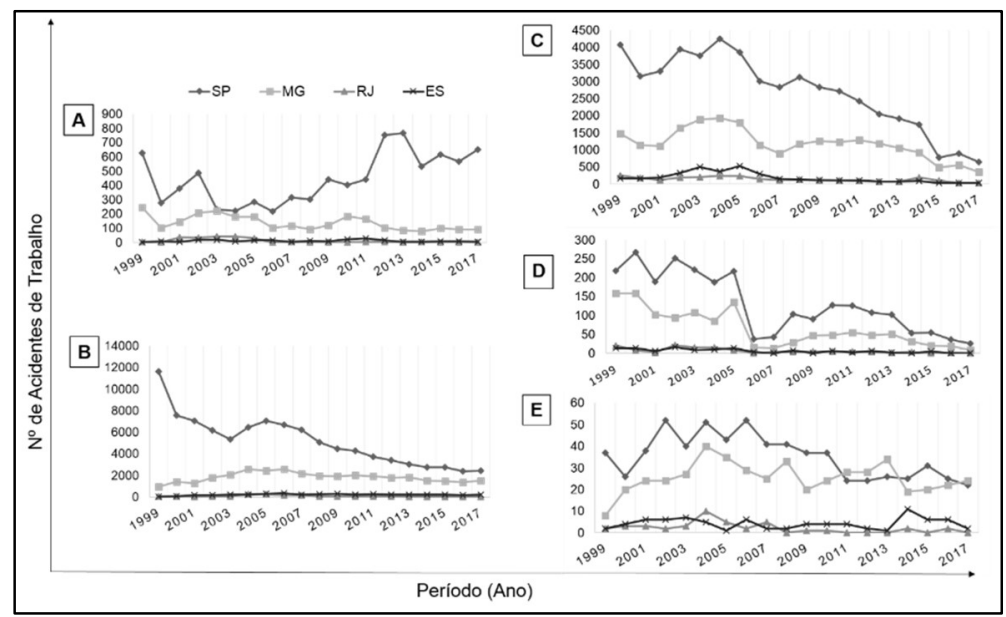

Figura 4: Evolução histórica dos acidentes de trabalho nos estados da região sudeste, entre 1999 e 2017, de acordo com as consequências (A - Assistência Médica; B - Incapacidade menos de 15 dias; C - Incapacidade mais de 15 dias; D - Incapacidade permanente; E - Óbitos).

Em São Paulo, a partir do ano em que a NR 31 entrou em vigor, foi observado um aumento crescente do número de acidentes com trabalhadores rurais que necessitaram somente de assistência médica e uma redução significativa dos acidentes com incapacidade por até 15 dias e incapacidade por mais de 15 dias, e o número de óbitos por ano (Figura 4). Apesar de ainda não corresponder ao ideal, os dados demonstram que está havendo uma redução na gravidade dos acidentes nas atividades agropecuárias de São Paulo. Já nos 
outros estados do Sudeste (MG, RJ e ES), a redução dos acidentes foi observada em todos os níveis de gravidade, ressaltando o RJ que não apresentou nenhum registro de óbito em 2008, 2011-2013, 2015 e 2017. Com a aprovação da NR31 empregados e empregadores puderam ter maior conhecimento das obrigações e responsabilidades de cada um. No entanto, mesmo passados 14 anos da criação dessa NR, os números de acidentes no meio rural ainda são preocupantes.

\section{CONCLUSÕES}

A análise da evolução dos acidentes de trabalho na agropecuária apresenta subsídios necessários para aumentar a eficiência de implantação da NR31 nas propriedades rurais. A aplicação eficiente dessa NR contribuirá para a melhoria na saúde, segurança e qualidade de vida dos trabalhadores, além de contribuir para a redução de impactos ambientais. Para isso, é importante que o empregador ofereça treinamentos adequados periodicamente e que sejam implementadas políticas de conscientização no ambiente laboral.

\section{REFERÊNCIAS}

ACOSTA, E. M.. Gestão de riscos ocupacionais do setor agrícola no município de Chapecó: Diagnóstico. Monografia (Especialização em Engenharia de Segurança do Trabalho) Universidade do Oeste de Santa Catarina, Chapecó, 2015.

ALMEIDA, W. F.. Trabalho agrícola e sua relação com saúde/doença. In: MENDES, R.. Patologia do Trabalho. Rio de Janeiro: Atheneu, 1995. p.487-516.

ALVARENGA, C. B.; VAL, V. L. P.; VAL JÚNIOR, N. A.; ZAMPIROLI, R.; RINALD, P. C. N.. Ocorrência de acidentes com máquinas agrícolas na região do cerrado de Minas Gerais. Revista Brasileira de Tecnologia Aplicada nas Ciências Agrárias, Guarapuava, v.10, n.3, p.27-36, 2017.

AMBROSI, J. N.; MAGGI, M. F.. Acidentes de trabalho relacionados às atividades agrícolas. Acta Iguazu, Cascavel, v.2, n.1, p.1-13, 2013.

BRASIL. Consolidação das Leis do Trabalho. Aprovada pelo decreto 5452, maio de 1943. Brasília: CLT, 1943.

BRASIL. Constituição da República Federativa do Brasil de 1988: Capítulo II dos Direitos Sociais. Art. 7. Brasília: DOU, 1988.

BRASIL. Decreto n. 4.552, de 27 de dezembro de 2002. Brasília: DOU, 2002.

BRASIL. Lei n. 5.889, de 05 de Junho de 1973. Brasília: DOU, 1973.
BRASIL. NR-31: Segurança e Saúde no Trabalho na Agricultura, Pecuária Silvicultura, Exploração Florestal e Aquicultura. Portaria GM n. 86, de 03 de março de 2005. Brasília: DOU, 2005.

BRAUND, W. E.; ALEXANDER, M.. Agricultural injuries: improving occupational safety. Medscape, 2007.

CEPEA. Centro de Estudos Avançados em Economia Aplicada. PIB Agro CEPEA-USP/CNA. CEPEA, 2017.

LOPES, C. V. A.; ALBUQUERQUE, G. S. C.. Agrochemicals and their impacts on human and environmental health: $a$ systematic review. Saúde debate, v.42, n.117, p.518-534, 2018. DOI: https://doi.org/10.1590/0103-1104201811714

RODRIGUES, V. L.; SILVA, J. G.. Acidentes de trabalho e modernização da agricultura brasileira. Revista Brasileira de Saúde Ocupacional. São Paulo, v.14, p.28-39, 1986.

SILVA, G. B.; BOTELHO, M. I. V.. O processo histórico da modernização da agricultura no Brasil (1960-1979). Revista de Geografia Agrária, Uberlândia, v.9, n.17, p.362-387, 2014.

SILVEIRA, C. A.; ROBAZZI, M. L. C. C.; MARZIALE, M. H. P.; DALRI, M. C. B.. Acidente de trabalho entre trabalhadores rurais e da agropecuária identificados através de registros hospitalares. Ciência, Cuidado e Saúde, Maringá, v.4, n.2, p.120-128, 2005.

A CBPC - Companhia Brasileira de Produção Científica (CNPJ: 11.221.422/0001-03) detém os direitos materiais desta publicação. Os direitos referem-se à publicação do trabalho em qualquer parte do mundo, incluindo os direitos às renovações, expansões e disseminações da contribuição, bem como outros direitos subsidiários. Todos os trabalhos publicados eletronicamente poderão posteriormente ser publicados em coletâneas impressas sob coordenação da Sustenere Publishing, da Companhia Brasileira de Produção Científica e seus parceiros autorizados. Os (as) autores (as) preservam os direitos autorais, mas não têm permissão para a publicação da contribuição em outro meio, impresso ou digital, em português ou em tradução. 